ORIGINAL ARTICLE

\title{
Patient profile and sleep abnormalities among the patients visiting a general physician in an urban slum area, Chittagong, Bangladesh
}

\section{Rajat Sanker Roy Biswas', Biplob Bhattacharjee ${ }^{2}$}

1. Dr. Rajat Sanker Roy Biswas, Resident Physician, Chattagram Maa Shishu O General Hospital

2. Dr. Biplob Bhattachariee, Assistant Professor, Chittagong Medical College, bsnrd@yahoo.com

\begin{abstract}
Background: In general practice, a variety of patients visit general physicians (GPs) for different clinical conditions. There is an influence of seasonal patterns on patient output. Among those, sleep disturbances is a common presenting complaint. Thus, the objective of this study was to find a pattern of GP visits for patients throughout a year with a special emphasis on frequency of insomnia and its characters.

Methods: A total of 977 patients visiting the researcher, who is also a GP in an urban slum area of Chittagong town, Bangladesh, in 2013 participated in the study. Information regarding sleep pattern and comorbidity in the form of acute and chronic disease was collected using a questionnaire. Necessary data was retrieved from those documents and analyzed using Microsoft Excel 2007.

Results: Of the 977 patients seen in the year 2013, there was a huge rush of patients from April to July. More female patients $(59.7 \%)$ visited GPs than the male $(40.3 \%)$ patients in which patients at both extremes of age ( $<12$ years, $35.31 \%$; $>60$ years, $21.3 \%)$ were the sufferers. Of all patients in whom most had short duration of sleep (53.8\%) and others had delayed onset of sleep (24.8\%) and early awakening $(21.4 \%)$, insomnia was found to be present in $17.7 \%$ (173 of 977 patients). Among 173 patients with insomnia, 67 (39\%) presented primarily with insomnia and other primary presenting medical problems, and $103(61 \%)$ had insomnia that was extracted on further query. Among all, 95 (54.91\%) had acute disease, 45 (26.01\%) had chronic disease, and 33 (19.07\%) had undetermined disease during the initial evaluation.
\end{abstract}

\section{Corresponding author}

\section{Dr. Rajat Sanker Roy Biswas}

Resident Physician,

Chattagram Maa Shishu O General Hospital,

Bangaladesh

Email: rajatbiswas76@yahoo.com

Contant: 8801819808433 
Conclusions: Sleep disorder is important comorbidity among patients with other medical conditions. Large-scale prevalence study with nationally representative sample is needed to find out the actual scenario of Bangladesh.

Keywords: Sleep, General physician, Slum area, Insomnia

\section{Introduction}

S leep is not merely a "time out" from our busy routines, it is essential for good health, mental and emotional functioning, and safety. Over the past few decades, there has been growing evidence to suggest that too little sleep and too much sleep are associated with adverse health outcomes, including total mortality, cardiovascular disease, type 2 diabetes, hypertension and respiratory disorders, obesity in both children and adults, and poor self-rated health ${ }^{1,2}$.

Most people experience at least an occasional night of poor sleep. However, certain individuals may be more vulnerable to long-standing difficulty. These include students; shift workers; travelers; and persons with acute stress, depression, or chronic pain or acute illness. Also, employees working long hours or multiple jobs may find their sleep less refreshing. The elderly also have frequent sleep problems, but inadequate sleep is not an inevitable part of the aging process. Those with acute disease or who had mental or physical illness are very much vulnerable to sleep disturbances.

Bangladesh is still an underdeveloped country and physicians are burdened with infectious and communicable disease $\mathrm{s}^{3}$. Thus, they have very little scope to deal with the noncommunicable disease such as sleep disturbances. This observational study was conducted to find a baseline information regarding pattern and prevalence with temporal variations of sleep disturbance among the patients visiting the general physicians (GPs) for different health-related problems.

\section{Materials and Methods}

\section{Study design}

This study was a cross-sectional, questionnaire survey conducted between January and December 2013 in a private primary care clinic in a slum area of Chittagong, Bangladesh. Primary care providers in Bangladesh can be broadly classified into private and government sectors.
An appropriate sampling technique was applied. A pilot study with 20 samples was conducted to see the validity of the questionnaire. An informed consent was obtained from every participating patient after explaining him/her the study procedure and objective.

\section{Sampling and enrollment criteria}

There is a huge gap in daily number of patients seen in government and private primary care chambers in Bangladesh. All patients in the chamber, aged from 1 year to 60 years and above, were invited to participate in the study. Those with serious illness, impaired memory, and mental illness; and women who were either pregnant or up to 6-week postpartum were excluded from the study, as they may not be able to provide accurate responses that may introduce bias to the study.

\section{Questionnaires}

The questionnaire was developed to see the frequency of insomnia; its nature whether early awakening, poor time, or delay to fall asleep; and data on age, gender, and comorbidities in terms of acute or chronic diseases. As it is a preliminary study on sleep problem in the setting of a developing country such as Bangladesh, questionnaire was not enriched further.

To evaluate their sleep pattern, respondents were asked about difficulty in falling asleep ( $>30 \mathrm{~min}$ to fall asleep), maintaining sleep ( $>3$ nighttime awakenings), and early morning awakening (waking between 2 and 5 a.m.). Regarding children of $<12$ years, response of mothers about night sleep pattern was considered to find the sleep problems.

The presence of any of the earlier-mentioned symptoms on at least 3 days in a week was considered to be insomnia, based on the International Classification of Sleep Disorders and the DSM-IV (Diagnostic and Statistical Manual of Mental Disorders) criteria ${ }^{4}$. 


\section{Results}

In the year in 2013 maximum number of patients presented to the GPs during the months of April to July. The total number was found to be 977 (Fig 1).

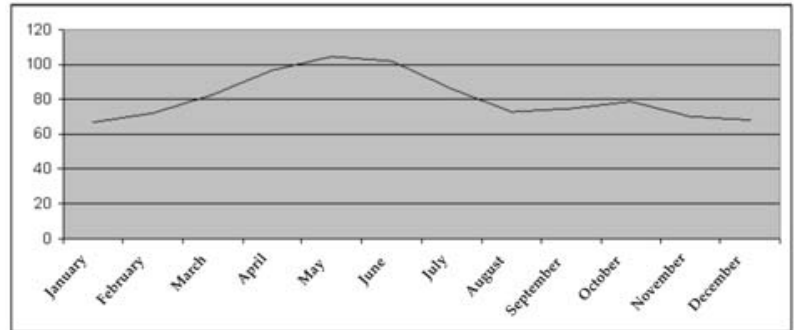

Figure 1: Month-wise profile of patients

In general sleep disorders were found to be more prevalent in the female patients than the males $(59.7 \%$ vs $40.3 \%)$ (Fig 2 ).

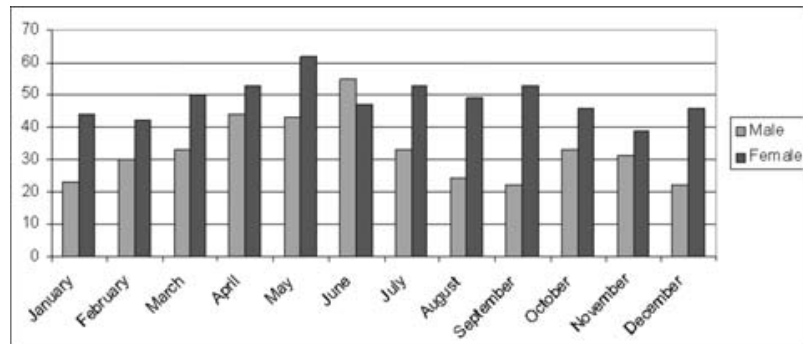

Figure 2: Gender-wise distribution of study patients.

The largest number of patients were found in extremes of age namely $<12$ years and $>60$ years old (Fig 3).

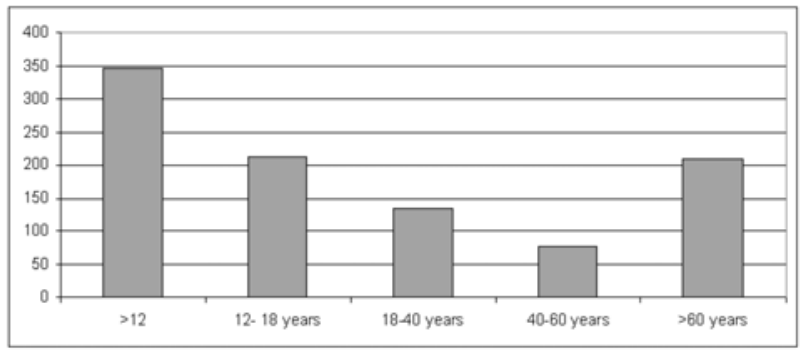

Figurec 3: Age group of the study patients.
The most common sleep disorder was found to be insomnia (17.7\%) (Fig 4).

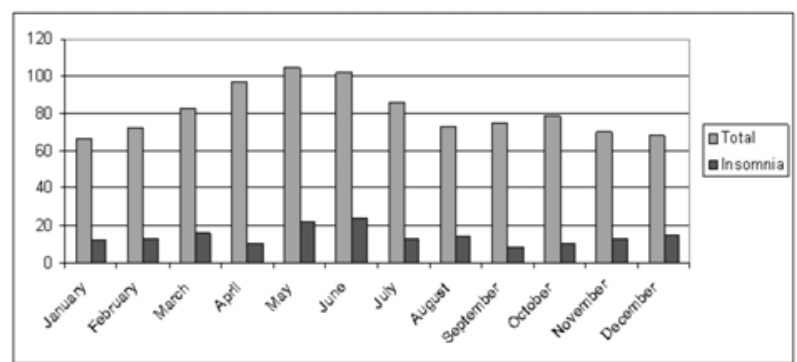

Figure 4: Month-wise distribution of insomnia in relation with total patients.

$24.8 \%$ of patients had delayed onset of sleep, $21.4 \%$ had early awakening and $53.8 \%$ had short duration of sleep (Fig 5).

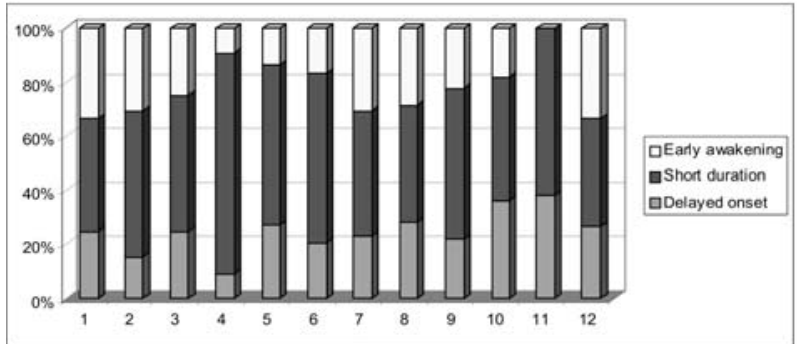

Figure 5: Type of insomnia in relation with total patients.

showing nature of co morbid disease with insomnia, where among 173 total patients of insomnia, 95(54.91\%) had acute disease, 45 (26.01\%) had chronic disease and $33(19.07 \%)$ had undetermined disease during the initial evaluation (Fig 6).

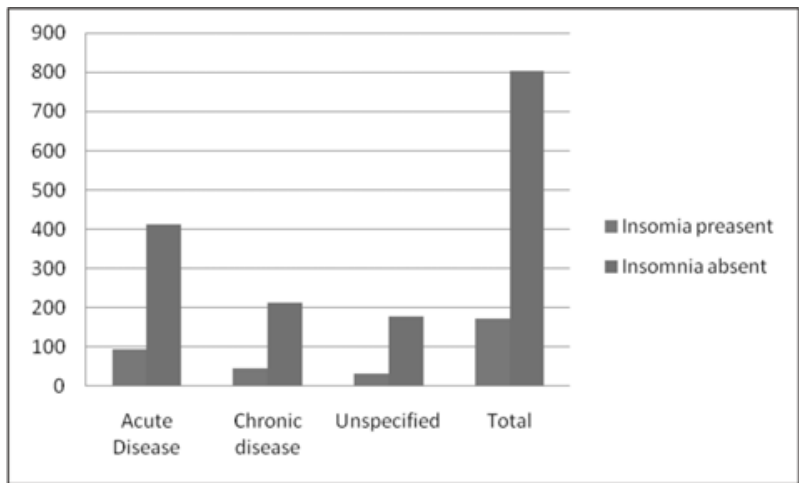

Figure 6: Relation of nature of comorbid disease with insomnia. 


\section{Discussion}

The study population was representative of the primary care patients in a selected area of Chittagong, Bangladesh. In our study, $17.7 \%$ of the patients who attended chamber due to any reason, experienced insomnia symptoms. Among them, 39\% had primary insomnia as a presenting complaint; and in remaining $61 \%$, the insomnia was observed on further questioning. Insomnia is a common public health problem with an estimated prevalence of $11 \%-50 \%$ in both Western and Asian general populations $s^{5-11}$. The wide variation of prevalence is due to differences in definitions of insomnia used, populations studied, and research methodology applied. This type of frequency in this study is probably due to the possible underlying physical and mental health problem that brought them to the primary care physician.

Primary care attendees presented with a variety of reasons for visits. Regarding nature of comorbid disease with insomnia in this study, 95 (54.91\%) had acute disease, $45(26.01 \%)$ had chronic disease, and 33 (19.07\%) had undetermined disease during the initial evaluation. The study conducted by Roth ${ }^{12}$ suggested that majority of those with insomnia seen in primary care clinics have comorbid conditions. Comorbid insomnia refers to insomnia related to certain medical conditions (psychiatric or medical disorders), medicines, and certain substances such as caffeine. The reason for visits and details of comorbidities was not considered for the sample population of this study. Shorter duration of sleep was the main type of sleep abnormality found among the study patients. As most of the patients had some types of physical problems for which they visited GP, presence of insomnia is expected.

In this study, March to August was found to be an important period when patient visit to GP increased in Bangladesh. Female patients, elderly, and children visited GP more often than adult male patients. Threshold for tolerance to ill health plays an important role in GP visits and patients at both extremes of age and female gender are vulnerable ${ }^{13,14}$. This scenario is common in India also ${ }^{15}$. Due to socio-economic reasons, the working class, which resides in slums, usually obtains medication directly from the pharmacist without consulting the doctors. Also, in Bangladesh almost every drug can be bought without prescription ${ }^{3}$.

This is an important study in primary care population in the private setting of Chittagong, Bangladesh, as it shows that insomnia is common and underrecognized. We were however unable to establish a causal link between insomnia and psychiatric disorders due to the cross-sectional design and the lack of clinical interview for diagnosing disorders in this study.

Indian Journal of Sleep Medicine (IJSM), Vol. 10, No. 1, 2015

\section{Conclusion}

Sleep disorder is an important comorbidity among patients with other medical conditions. A large-scale prevalence study with nationally representative sample is needed to find out the actual scenario of Bangladesh.

\section{References}

1. Banks S, Dinges DF. Behavioral and physiological consequences of sleep restriction. J Clin Sleep Med 2007;35:519-28.

2. Cappuccio FP. D'Elia L, Strazzullo P, Miller MA. Sleep duration and all-cause mortality: a systematic review and meta-analysis of prospective studies. Sleep $2010 ; 33(5): 585-92$.

3. World Health Organization. Global Health Observatory (GHO) Data. Bangladesh Country Profile. Available at http:/ /www.who.int/gho/countries/bgd/country_profiles/en/ (retrieved 1-1-2015).

4. American Psychiatric Association. Diagnostic and Statistical Manual of Mental Disorders, 4th edn. Washington, DC, American Psychiatric Association, 1994.

5. Ustun TB, Privett $M$, Lecrubier Y. Form, frequency and burden of sleep problem in general health care: a report from the WHO collaborative study on psychological problem in general health care. Eur Psychiatry 1996;1 1 (Supp 1):5S-10S.

6. Ohayon $\mathbf{M M}$. Epidemiology of insomnia: what we know and what we still need to learn. Sleep Med Rev $2002 ; 6(2): 97-111$.

7. Groeger JA, Zijlstra FR, Dijk DJ. Sleep quantity, sleep difficulties and their perceived consequences in a representative sample of some 2000 British adults. J Sleep Res 2004;13(4):359-71.

8. Kim K, Uchiyama M, Okawa M, Liu X, Ogihara R. An epidemiological study of insomnia among the Japanese general population. Sleep 2000;23(1):41-7.

9. Yeo BK, Perera IS, Kok LP, Tsoi WF. Insomnia in the community. Singapore Med J 1996;37:282-4.

10. Li RH, Wing YK, Ho SC, Fong S. Gender differences in insomnia: a study in the Hong Kong Chinese population. $J$ Psychosom Res 2002;53:601-9.

11. Ohayon MM, Hong SC. Prevalence of insomnia and associated factors in South Korea. J Psychosom Res $2002 ; 53: 593-600$

12. Roth T. Comorbid insomnia: current directions and future challenges. Am J Manag Care 2009;15(Suppl):S6-S13.

13. Patel SR, Ayas NT, Malhotra MR, White DP, Schernhammer $\mathrm{ES}$, Speizer FE, et al. A prospective study of sleep duration and mortality risk in women. Sleep 2004;27:440-4.

14. Ayas NT, White DP, Manson JE, Stampfer MJ, Speizer FE, Malhotra A, et al. A prospective study of sleep duration and coronary heart disease in women. Arch Intern Med $2003 ; 1632: 205-9$.

15. Shah N, Bang A, Bhagat A. Indian research on sleep disorders. Indian J Psychiatry 2010;52(Suppl 1):S255-9. 